Musées, Patrimoine et Culture scientifiques et techniques

$129 \mid 2010$

mai - juin 2010

\title{
Patrimoine contemporain et culture scientifique et technique
}

Contemporary heritage and scientific and technical culture

\section{Catherine Cuenca}

\section{OpenEdition}

\section{Journals}

Édition électronique

URL : http://journals.openedition.org/ocim/153

DOI : 10.4000/ocim.153

ISSN : 2108-646X

Éditeur

OCIM

Édition imprimée

Date de publication : 1 mai 2010

Pagination : 21-27

ISSN : 0994-1908

Référence électronique

Catherine Cuenca, "Patrimoine contemporain et culture scientifique et technique », La Lettre de l'OCIM [En ligne], 129 | 2010, mis en ligne le 01 mai 2012, consulté le 21 avril 2019. URL : http:// journals.openedition.org/ocim/153; DOI : 10.4000/ocim.153 


\section{Patrimoine contemporain et culture scientifique et technique}

Catherine Cuenca *

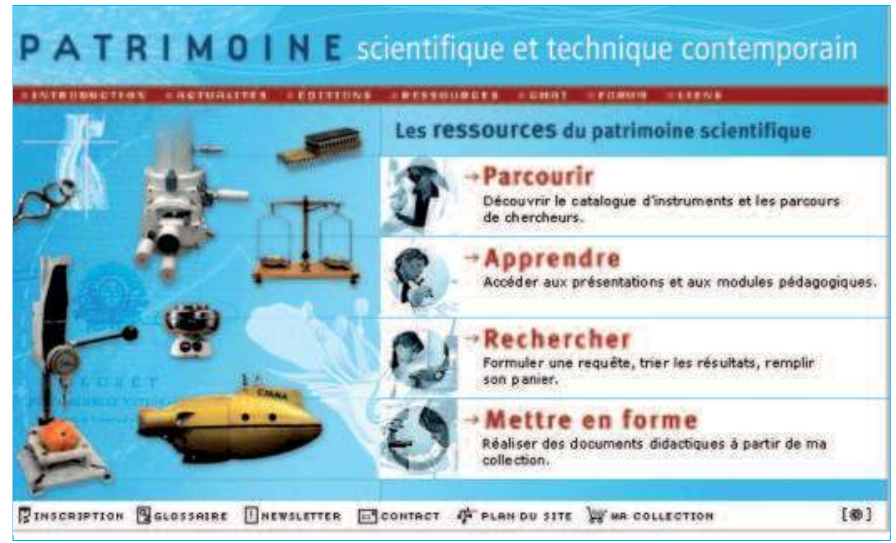

Page d 'accueil du site Internet national de la Mission, www. patstec.fr (C) Université de Nantes-Mission Patstec
Responsable de la Mission nationale du musée des Arts et Métiers, l'auteur présente les objectifs et les premières réalisations de cet outil mis en place pour inventorier, sensibiliser à la sauvegarde, conserver et valoriser le patrimoine scientifique et technique de ces 50 dernières années, mettant en particulier l'accent sur l'organisation régionale du réseau constitué et son ouverture à l'échelle européenne.

Cet article retrace brièvement quatorze années d'expériences de sauvegarde, d'inventaires et de valorisation du "patrimoine scientifique et technique contemporain » à la fois matériel, constitué par les objets construits et utilisés au cours des cinquante dernières années et immatériel sous forme de témoignages de chercheurs (et de leurs équipes) dans les établissements universitaires et de recherche et dans les laboratoires des entreprises. Un premier programme a été mené au sein de l'université de Nantes, un second s'est étendu aux niveaux des organismes de la région des Pays de la Loire. Ces deux initiatives ont précédé la création d'une Mission nationale de sauvegarde du patrimoine scientifique et technique contemporain confiée, en 2003, au musée des Arts et Métiers par le ministère de la Recherche.

Nous présenterons plus particulièrement les objectifs et les résultats obtenus de 2003 à 2009 par la Mission nationale. Les nombreuses actions menées s'inscrivent dans une prise de conscience de l'importance de ce patrimoine contemporain. En effet, la sauvegarde du matériel et des savoir-faire des laboratoires de la 


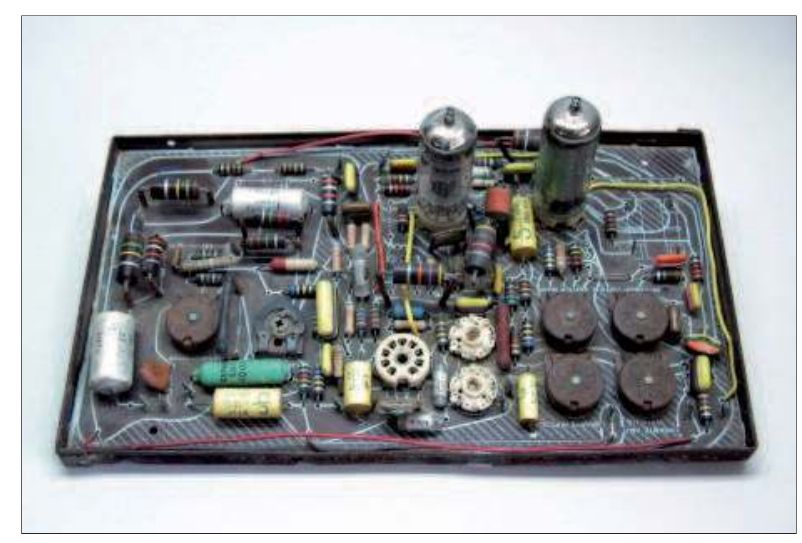

Circuit imprimé, 1950-1975, université de Nantes () Université de Nantes-Mission Patstec

recherche publique et privée largement tournés vers l'innovation et l'avenir, représente un enjeu important pour le développement de la culture scientifique et technique actuelle et future.

\section{Un bref rappel historique}

L'attrait pour les œuvres d'art, les curiosités de la nature et les instruments scientifiques et techniques, réunis pour satisfaire le goût esthétique, la curiosité intellectuelle et la connaissance va s'étendre de la Renaissance au siècle des Lumières dans toute l'Europe ${ }^{(1)}$. Ainsi, apparaissent les premiers « cabinets de curiosités ", constitués à partir de spécimens de Sciences naturelles, d'Ethnologie et de tous objets remarquables ramenés des expéditions scientifiques, puis les cabinets de Physique et de Mécanique. Les collections éléments de prestige des cours royales, princières et ecclésiastiques sont également le fait des élites professionnelles - avocats ou médecins. Avec l'ouverture des premiers musées publics à la fin du XVIII e siècle, il s'agit dorénavant de conserver et de montrer les objets au nom de la science expérimentale et de son utilité sociale.

Peu à peu, ces regroupements d'objets deviennent le support de « la connaissance et de l'instruction». À la Révolution, après une période de destruction massive de tout ce qui représente les privilèges abolis, la volonté est de rendre ces connaissances accessibles aux citoyens. L'abbé Grégoire défendra la construction du Conservatoire des Arts et Métiers en 1794 dans lequel il ne suffit pas de montrer et d'expliquer mais également de démontrer et d'enseigner. Dans la deuxième moitié du XIXe siècle, les expositions universelles présentent les réalisations industrielles des différentes nations. Elles témoignent du progrès

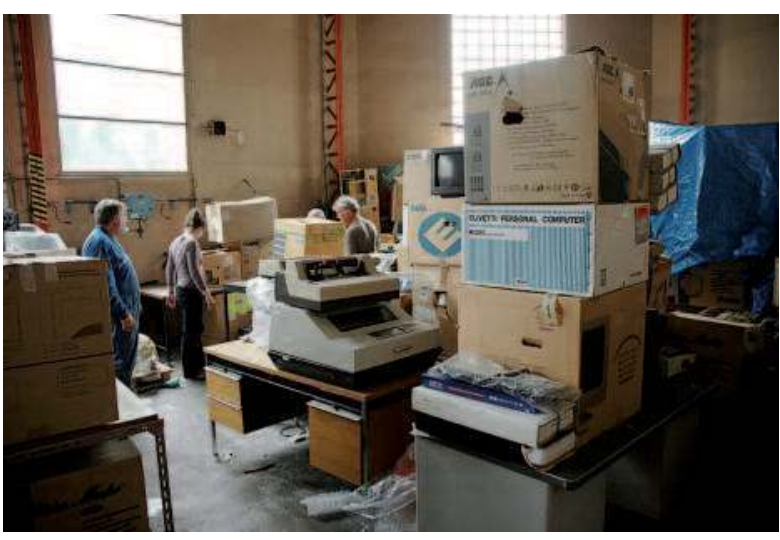

Le tri des objets avec le groupe « patrimoine science» de l'université de Nantes () Université de Nantes-Mission Patstec

technologique survenu au cours de la Révolution industrielle. Les objets présentés dans ces expositions sont à l'origine de la naissance des grands musées d'art et d'industrie, qui se transforment progressivement en musées des sciences et des techniques ${ }^{(2)}$. Les traces matérielles et immatérielles de cette mémoire scientifique et technique, accumulées et transmises, constituent aujourd'hui une partie importante de notre culture.

Quelle histoire du XXe siècle notre société lègue-telle au XXIe siècle ? L'évolution des sciences et des technologies a été très rapide au cours des cinquante dernières années donnant lieu à des transformations spectaculaires. De nombreux chercheurs qui ont participé à la création et à la dynamique des laboratoires de recherche dans les années 1960 quittent actuellement la vie professionnelle, et les instruments scientifiques utilisés dans leurs travaux disparaissent, effaçant une mémoire irremplaçable de ce demi-siècle d'évolution scientifique et technique.

\section{En 1996, un programme " Mémoires de l'innovation technologique " à I'université de Nantes}

Sous l'impulsion de la Direction de l'information scientifique, des technologies nouvelles et des bibliothèques du ministère de la Recherche et de l'Éducation nationale, de la "Mission musées » de ce même ministère et avec le soutien du président de l'université de Nantes, une mission de trois ans m'a été confiée, afin de "repérer » le patrimoine scientifique et technique de cette université et de mener des actions de valorisation auprès du public. Suite aux entretiens avec les doyens et les responsables des UFR de l'université, la principale ligne 
directrice retenue a été de travailler sur la problématique du patrimoine scientifique et technique des quarante à cinquante dernières années.

Des objets sont alors répertoriés en vue de leur sauvegarde. Il s'agit d'instruments utilisés et parfois créés par les chercheurs depuis les années 1950. Ces objets témoignent de la recherche et de l'enseignement supérieur contemporains - instruments scientifiques, documents associés tels que les notices d'emploi, les cahiers de laboratoire, les catalogues techniques. Sans action de sensibilisation et de sauvegarde, ces matériels, souvent réformés dans les laboratoires et les services, tendent à disparaître à jamais sans laisser de traces. Au cours de ce premier repérage, plusieurs questions se sont posées relatives aux modalités et aux critères de sélection et de tri ainsi qu'aux informations susceptibles de replacer les objets dans leur contexte d'origine. Toutes ces interrogations renvoient nécessairement à une réflexion plus générale sur les nouvelles catégories ou typologies d'objets constituées.

\section{Un programme élargi aux structures universitaires régionales}

En 1999, le programme de sauvegarde a concerné l'ensemble des universités et organismes de recherche de la région des Pays de la Loire, avec le soutien essentiel du Délégué régional à la recherche et à la technologie en Pays de la Loire (3). Ce programme régional, intitulé « Mémoires de l'innovation scientifique et technologique du XXe siècle » est porté par l'université de Nantes et un Groupement d'Intérêt Public Régional, Atlantech, qui offrait la structure adéquate à la fois pour la mobilisation des acteurs régionaux et pour le soutien administratif nécessaire au projet. De plus, un comité scientifique et un comité de pilotage ont été mis en place permettant d'échanger et de valider régulièrement l'avancement du projet, s'appuyant sur un réseau de correspondants scientifiques et techniques (chercheurs, universitaires, industriels, techniciens) dans la région des Pays de la Loire ${ }^{(4)}$.

Une composante multimédia est ajoutée à réalisation de l'inventaire et de la sauvegarde en vue de donner une visibilité immédiate au projet en utilisant les possibilités offertes par les nouvelles technologies. Dans cette orientation multimédia, trois DVDROM Histoires de chercheurs, ont été réalisés portant sur dix-sept thématiques de recherche développées dans la région et destinés à un large public - lycéens, étudiants, enseignants, muséologues et grand public. Aujourd'hui, de nouveaux concepts ont été développés autour, cette fois, « d'histoires de laboratoires » (5).

\section{La création de la Mission nationale de sauvegarde : objectifs et méthodes}

Lors d'un colloque en 2001 à Orsay, et une rencontre avec le directeur du musée des Arts et Métiers (6) - soucieux de développer une collection portant sur cette période de la deuxième moitié du XXe siècle dans son établissement - mettre en œuvre une politique de sauvegarde de ce «nouveau patrimoine » au plan national, est apparu comme une nécessité. Dans cette optique, il semblait très pertinent de transposer et de généraliser à l'échelle nationale les méthodes expérimentées au niveau d'un organisme puis d'une région. En 2003, une Mission nationale de sauvegarde du patrimoine scientifique et technique est confiée au directeur du musée des Arts et Métiers par le ministre de la Recherche, les compétences et outils développés en Pays de Loire sont alors mis à la disposition de la Mission nationale dont les principaux objectifs sont définis :

- sensibiliser les organismes d'enseignement supérieur et de recherche et les entreprises à la sauvegarde du patrimoine scientifique et technique contemporain ; - développer un réseau national de sauvegarde réaliser un inventaire national et valoriser le patrimoine de la recherche et de l'industrie, en assurant un rôle de conseil et d'expertise dans le domaine visible pour tous les publics, dans un site Internet ;

- susciter des initiatives régionales, en accompagnant la mise en œuvre du programme dans les régions.

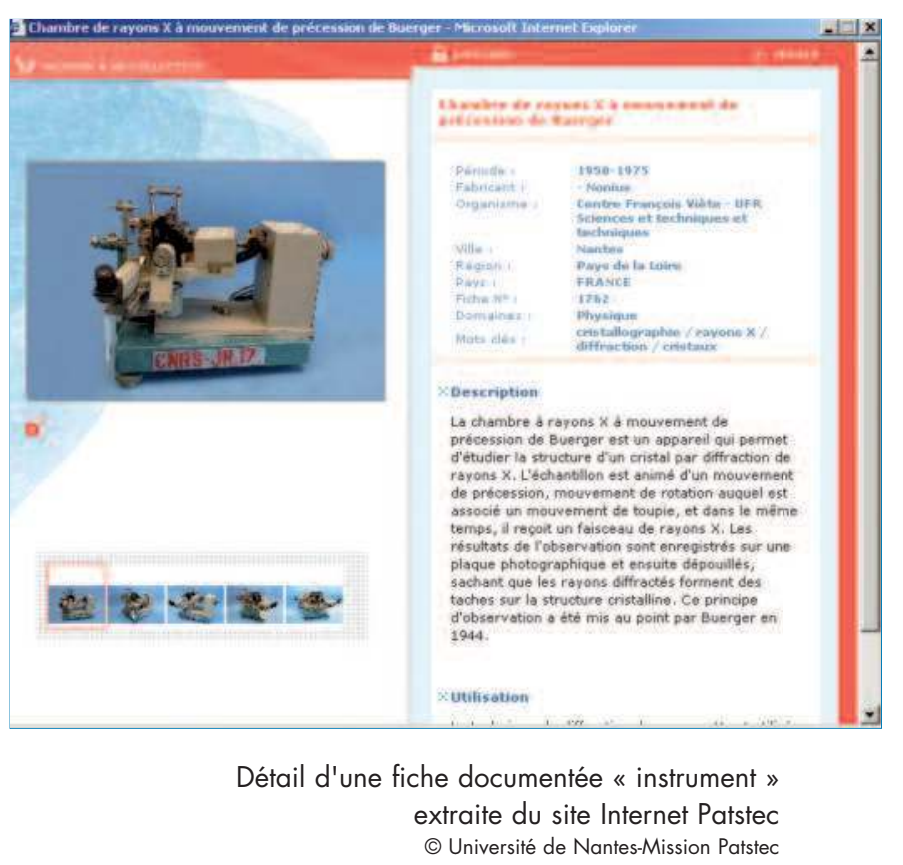


Ces actions de sauvegarde concernent, d'une part, les objets témoins de l'enseignement et de la recherche publique et privée, - de l'instrument au produit industriel, et la documentation associée - et, d'autre part, les témoignages et les savoir-faire des hommes et femmes de la recherche qui ont inventé ou utilisé ces objets. Ce "patrimoine ", dans sa grande diversité, constitue un outil privilégié pour permettre à nos concitoyens de se familiariser avec les savoirs, les techniques et les innovations. Sa mise en valeur peut également susciter chez les jeunes des vocations dans les domaines de la science, de la recherche et de la technologie ${ }^{(7)}$.

Pour mener à bien l'ensemble des objectifs de la mission, est créé au sein du musée des Arts et Métiers un dispositif composé d'une cellule de coordination nationale, d'un conseil scientifique, d'un observatoire de ce patrimoine scientifique et technique ainsi que des outils multimédia - base de données nationale et site Internet. La base nationale accueille des données réunies et choisies par les partenaires régionaux du réseau national : fiches d'inventaires d'objets, interviews et vidéos de chercheurs, animations pédagogiques. Ces ressources sont ensuite transférées dans le site Internet (www.patstec.fr). L'ensemble constitue un « centre de ressources » sur le thème (actuellement plus de 7300 fiches, 20000 photographies, vidéos et animations) et un outil pédagogique de culture scientifique et technique, grâce aux technologies développées dans le site Internet, notamment pour l'enseignement et la formation.

\section{Le réseau national "Patstec ": résultats en 2010}

Le réseau comprend aujourd'hui quatorze régions partenaires : Aquitaine, Auvergne, Alsace, Bretagne, Bourgogne, Champagne-Ardenne, Franche-Comté, Haute-Normandie, Languedoc-Roussillon, Lorraine, Midi-Pyrénées, Nord-Pas-de-Calais, et Rhône-Alpes, en plus de lîle-de-France et des Pays de la Loire. À la participation de ces régions s'ajoute celle de partenaires industriels tels : Essilor, Fondation EDF, Michelin, Schneider-Électric, de grands organismes de recherche européens (CERN) et, depuis 2010, le CNRS et Météo France. Chaque mission régionale est coordonnée par un chef de projet rattaché soit à une institution universitaire, soit à un musée de sciences, soit à une délégation régionale du Cnam. Les orientations de ces missions régionales sont suivies par un comité de pilotage composé de membres de différents départements universitaires, organismes de recherche et grandes écoles ; mais aussi des acteurs locaux de la culture, avec l'appui des DRRT, DRAC et collectivités. Dans certaines régions, ces actions peuvent s'institutionnaliser et aller jusqu'à la création d'unités, antennes ou musées scientifiques et techniques.

Une journée professionnelle nationale permet chaque année de faire le point sur l'avancement des inventaires et les actions de valorisation et de formation réalisées dans les régions partenaires. Cette journée est aussi l'occasion de sensibiliser de nouveaux partenaires universitaires et industriels à la nécessité de la sauvegarde et à l'intérêt de rejoindre le réseau. Des ateliers de travail réunissent les chefs de projet deux fois par an pour mutualiser les pratiques et la réflexion et déterminer des thèmes ou des points particuliers à traiter dans le cadre de journées d'étude et de colloque ${ }^{(8)}$. Leélaboration de critères scientifiques et historiques, un questionnement sur l'évolution des instruments et la notion d'innovation sont des éléments déterminants pour ce choix. Ainsi, un trait marquant du XXe siècle est l'arrivée de l'informatique et des nouvelles technologies de l'information dans la recherche, l'industrie et la vie quotidienne qui ont radicalement bouleversé les habitudes de chacun.

Des groupes de travail ont été constitués pour mener une réflexion sur les différentes questions posées par cette nouvelle problématique patrimoniale. Comment sélectionner les objets représentatifs des cinquante dernières années ? Avec quels critères effectuer le tri ? Comment replacer les objets dans leur contexte ? Un premier objectif est d'élaborer des listes d'instruments scientifiques référents, reflétant la recherche scientifique des soixante dernières années, par discipline ou thématique.

Par ailleurs, la constitution de ce «patrimoine » soulève de nombreuses questions à caractère juridique. Les objets repérés dans les laboratoires ont en effet des origines et des statuts variés. Pour répondre à ces interrogations, un guide juridique a été édité par Marie Cornu, directrice du CECOJI (CNRS), spécialiste du droit du patrimoine culturel, avec la participation des membres du réseau de la mission et le soutien du ministère de la Recherche et de l'Éducation nationale ${ }^{(9)}$. En 2008, afin de mettre en relation les professionnels du patrimoine et les spécialistes du milieu juridique, la Mission nationale s'est associée au CECOJI (Centre d'Études sur la Coopération juridique internationale) et à l'université de Sceaux pour l'organisation d'un colloque sur « Les aspects juridiques des collections scientifiques et techniques » au musée des Arts et Métiers. 


\section{Coup de plumeau sur le patrimoine scientifique toulousain}

C'est un fait qui n'est plus à prouver, la majorité du patrimoine scientifique contemporain universitaire s'entasse dans les caves et greniers et s'enfouit sous une couche de poussières. À l'abri des regards, ces instruments sauvés un jour «parce qu'ils pourraient resservir » s'effacent progressivement de la mémoire de ceux qui les ont entreposés et de leurs successeurs. Jusqu'au jour où... une équipe du réseau PATSTEC s'annonce et ose le coup de plumeau ! Les convaincus applaudissent, les perplexes disent pourquoi pas, les autres espèrent être débarrassés un jour de ces vieilleries... Mais quoiqu'il en soit l'équipe est généralement bien reçue ; les universités et écoles d'ingénieurs s'accordent sur le fait qu'elles doivent s'organiser pour s'occuper de leur patrimoine scientifique (et ce quelles qu'en soient les raisons et finalités !).

En 2007, une étape importante est franchie : dans le cadre de la restructuration de la Branche d'Activités Professionnelles F (BAP Information, Documentation, Culture, Communication, Edition, TICE), qui inclut des profils de poste Patrimoine de tous les corps de métiers, de l'adjoint technique à l'ingénieur de recherche, l'université de Toulouse (Pôle de Recherche et d'Enseignement Supérieur) ouvre la voie avec la création d'un poste de chargé de collections muséales, responsable du patrimoine scientifique contemporain. Ce poste a été créé en réponse à un besoin unanime de ses membres fondateurs et associés. Un besoin qui a été exprimé et entendu grâce au dynamisme et à l'implication du comité de pilotage de la mission de sauvegarde de Midi-Pyrénées. En effet, dès sa création en 2004, de remarquables énergies se sont mobilisées. Au côté du musée des Arts et Métiers, les collectivités, le CCSTI Science Animation et les universités au travers de leurs représentants engagés se réunissent, débâtent et militent sans s'essouffler tous les 4 mois environ depuis maintenant 5 ans. La sauvegarde du patrimoine scientifique trouve ainsi une cohérence et pertinence en se positionnant clairement au sein d'une structure fédérative au niveau régional.

Outre l'intérêt évident de mutualisation des moyens, la prise en charge interuniversitaire de ce patrimoine accroît la valeur ajoutée de chaque instrument préservé. L'instrument perçu en premier lieu comme détenteur de « son » histoire liée aux actions passées de « son » laboratoire, devient clairement témoin de l'histoire de « sa » discipline au sein de multiples projets pluridisciplinaires et impliquant plusieurs partenaires au niveau régional. En parallèle du travail d'inventaire, la Mission MidiPyrénées s'est, depuis sa création, donnée pour objectif

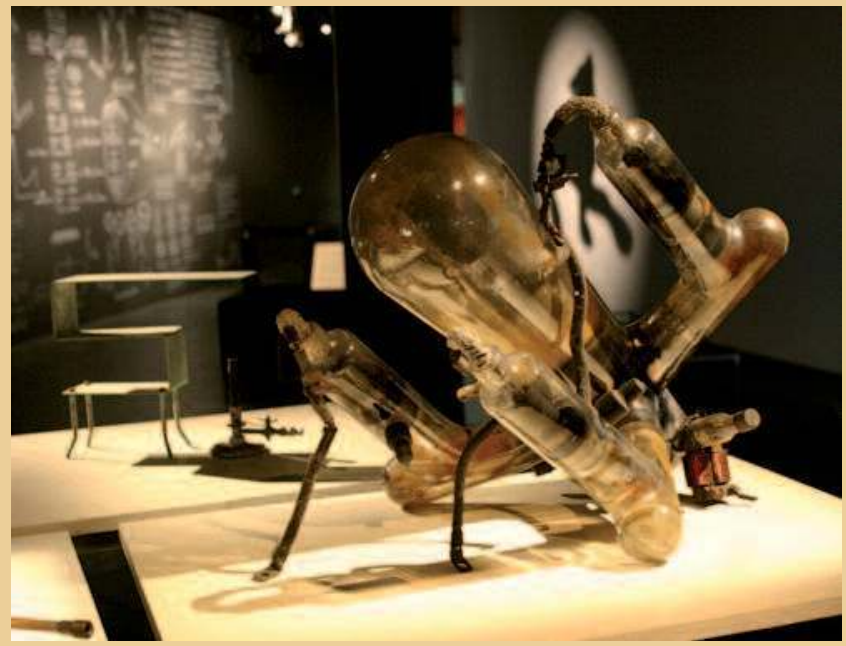

Redresseur triphasé à vapeur de mercure, ENSEEIHT (c) Mission sauvegarde du patrimoine scientifique et technique contemporain MIP

prioritaire d'afficher son patrimoine en le dépoussiérant au sens propre et au sens figuré. L'énergie dépensée dans cette valorisation (expositions, site Internet, newsletter Le Plumeau, jeu concours...) nous a permis de convaincre et ainsi de pérenniser la Mission avec la création d'un poste dédié de l'université de Toulouse - PRES.

Cependant, beaucoup de travail reste à faire. Quels que soient l'organisation choisie, les bonnes volontés et les moyens humains et financiers déployés, il faut toujours conserver l'ambition de convaincre les plus dubitatifs. En Midi-Pyrénées, il nous faut renforcer une équipe qui permettra de poursuivre un travail d'inventaire approfondi. Nous espérons également impulser des sujets de recherche en histoire des sciences et apporter une dimension patrimoniale à la formation des étudiants et des doctorants. Aujourd'hui, le patrimoine scientifique universitaire toulousain n'est pas laissé pour compte. Le contexte est favorable et nous permet depuis janvier 2010 de développer une politique de culture scientifique, elle-même interuniversitaire et conçue en concertation et de façon cohérente et coordonnée avec les collectivités, une politique dans laquelle le patrimoine scientifique universitaire, plus ou moins récent, est l'un des principaux points d'ancrage.

AnNe-Claire Jolivet

Responsable de la mission sanvegarde du patrimoine scientifique et technique contemporain de Midi-Pyrénées, Pôle de Recherche et d'Enseignement supérieur, université de Toulouse 


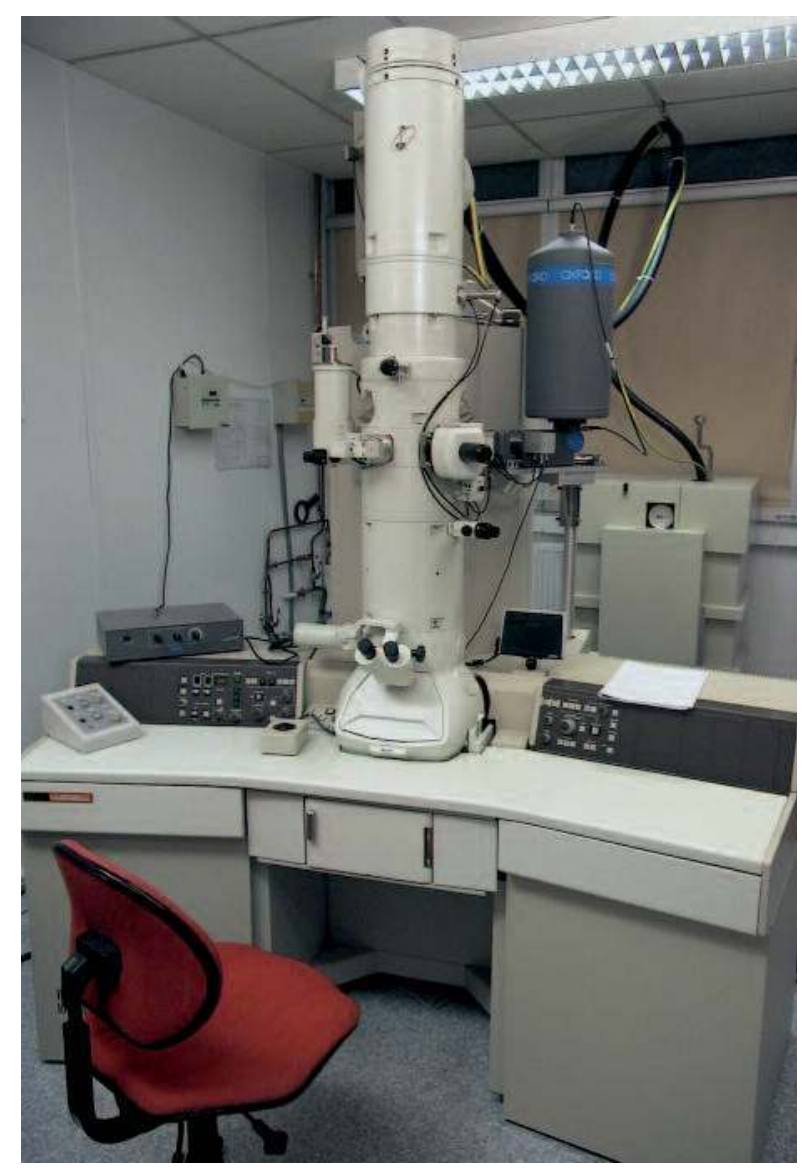

Microscope électronique à transmission, 1975-2000, JEOL, université d'Angers ๑) Université de Nantes-Mission Patstec

\section{Vers une ouverture européenne}

Plus largement, la mission nationale inscrit sa réflexion et ses actions dans le cadre d'une coopération internationale au sein de réseaux d'experts déjà existants, la SIC (Symposium International Commission) (10), Universeum (11), l'UMAC (University Museums and Collections) (12). Dans cette même perspective, une journée d'étude sur le patrimoine scientifique et technique contemporain a été organisée en 2006 par la Mission du musée des Arts et Métiers, réunissant des responsables de collections de grands musées de sciences et de techniques européens (Leonardo da Vinci Museum à Milan, Deutsches Museum à Munich, Science Museum à Londres, Museum à Leyde, National Science and Technology Museum à Madrid, Smithsonian Institution aux ÉtatsUnis...). Cette mise en commun des expériences s'est poursuivie, en mars 2008, lors du colloque organisé par la Mission au musée des Arts et Métiers sur le thème du « Patrimoine scientifique et technique, culture et société ». Les actes de ces deux manifestations donnent lieu à un ouvrage, publié en $2010^{\text {(13) }}$.
Ces différents échanges et collaborations ont débouché sur la volonté de mettre en commun les expériences dans le domaine de la sauvegarde du patrimoine scientifique et technique contemporain au niveau européen et un consortium avec une quinzaine de partenaires a été créé.

Cet objectif, soutenu fortement par le directeur du musée des Arts et Métiers, s'est concrétisé en novembre 2008 par l'organisation, à Milan, avec le musée des Sciences et Techniques Leonard de Vinci d'une première rencontre en vue d'engager avec plusieurs autres pays partenaires un programme européen dans ce domaine ${ }^{(14)}$. En parallèle, une rencontre a eu lieu avec les organismes universitaires et culturels de la région de Milan, pour lancer la première journée nationale d'un programme de sauvegarde, la mission nationale Patstec a apporté conseils et soutien. Un partenariat est également engagé entre le musée des Arts et Métiers et la maison de la Métallurgie et de l'Industrie et avec l'université de Liège - en particulier avec le centre d'Histoire des Sciences et des Techniques, un exemple de collaboration est la création d'un réseau francophone sur le patrimoine informatique.

La constitution de ce patrimoine - matériel et immatériel - soulève toujours de nombreuses questions relatives à diverses disciplines scientifiques et à des pratiques patrimoniales spécifiques qui, de ce fait, nécessitent parfois des solutions nouvelles. « Le contexte culturel et social actuel nécessite de garder les témoignages de la science et de la technique pour sensibiliser les jeunes à s'engager dans les carrières scientifiques, pour renforcer le lien entre les sciences et la société et faire connaître à un large public les innovations des soixante dernières années » (15). Ce sont ces objectifs que la Mission nationale du musée des Arts et Métiers souhaite atteindre par les opérations menées depuis plusieurs années.

\section{Notes}

(1) Ballé, C. et Poulot, D. Musées en Europe, une mutation inachevée. Paris : La Documentation française, 2004.

(2) Ballé, C. «Les sciences et techniques, une tradition muséale », La Revue du Cnam, n51-52, avril 2010.

(3) Yves Thomas est alors professeur d'université et délégué régional à la recherche et à la technologie-Pays de la Loire.

(4) Ballé, C. (ed.), Cuenca, C. et Thomas, Y. Le patrimoine scientifique et technique contemporain. Un programme de sauvegarde en Pays de la Loire. Paris : L'Harmattan, 2005.

(5) Thomas, Y. et Cuenca, C. L'apport des technologies de l'information et de la communication (TIC) à la sauvegarde du patrimoine scientifique 
et technique contemporain (Patstec), Documents pour l'histoire des techniques, $\mathrm{n}^{\circ} 18$, décembre 2009 .

(6) Daniel Thoulouze, alors directeur du musée des Arts et Métiers, Cnam

(7) Thoulouze. D. Le patrimoine scientifique et technique contemporain, La Revue du Cnam, n43-44, 2005, pp. 4-9.

(8) Ainsi, des journées d'études thématiques ont porté sur le patrimoine scientifique et technique et industriel, les multimédia et la modélisation numérique. Deux colloques ont été organisés par la mission en 2008 et donnent lieu à des publications : Patrimoine scientifique et technique, culture et société et Les aspects juridiques des collections scientifiques et techniques.

(9) Rainette, C., Cornu, M. (dir.) et Wallaert, C. (dir.). Guide juridique sur le patrimoine scientifique et technique. Paris : L'Harmattan, 2008.
(10) Paolo Brenni, chercheur au CNR à Florence, est le président de la commission instrumentation de la SIC.

(11) Universeum est un réseau européen de musées et collections universitaires, créé en 2000

(12) L'UMAC est une section de l'ICOM créée en 2001.

(13) Ballé, C. et Cuenca, C. (eds) Le patrimoine scientifique et technique: un enjeu contemporain. Paris : La Documentation française, à paraître en 2010 .

(14) Serge Chambaud, directeur de la Culture scientifique et du musée depuis 2007

(15) Serge Chambaud, conférence dans le cadre du colloque « Gestion et diffusion des collections universitaires », Symposium Québec, 25 et 26 mars 2010 . 